\title{
El adulto mayor en la sociedad
}

\section{(c) (i) () (2)}

The elderly in society

Adriana Mederos González. ${ }^{1}$ \& Lic. Juan Francisco Rojas Puig. ${ }^{2}$

\begin{abstract}
.
The third age is a stage of life in which the human being elaborates a reflection of what he has achieved in his life trajectory, as well as the opportunities that have been missed or simply have been postponed. It should be noted that biological and psychological disorders are present at this stage. That is, you begin to have problems with vision, hearing, speech, balance, and memory loss.
\end{abstract}

Keywords: Elderly, biological and psychological disorders.

\section{Resumen.}

La tercera edad es una etapa de la vida en la que el ser humano elabora una reflexión de lo que ha logrado en su trayectoria de vida, así como de las oportunidades que se han dejado pasar o simplemente se han postergado. Cabe señalar que en esta etapa se hacen presentes los trastornos biológicos y psicológicos. Es decir, se comienza a tener problemas con la vista, la audición, el habla, el equilibrio y la pérdida de memoria.

Palabras claves: Tercera edad, trastornos biológicos y psicológicos.

\footnotetext{
${ }^{1}$ Ciencias Médicas Mayabeque, Mayabeque, Cuba, amederos@infomed.sld.cu 0002-7353-0148

https://orcid.org/0000-

${ }^{2}$ Ciencias Médicas Mayabeque, Mayabeque, Cuba, jrojas@infomed.sld.cu 7353-0149
} 


\section{Introducción.}

La Tercera edad es un término antropo-social que hace referencia a la población de personas mayores o ancianas. En esta etapa el cuerpo se va deteriorando y, por consiguiente, es sinónimo de vejez y de ancianidad. Entre las enfermedades asociadas a la vejez se encuentra Alzheimer, artrosis, diabetes, cataratas, osteoporosis y la hipertensión arterial.

\section{Objetivos}

- Demostrar los métodos para combatir la discriminación del adulto mayor

- Explicar las acciones de prevención de la HTA y el sistema de ejercicios para hipertensos de la tercera edad.

\section{Desarrollo}

Las diferentes denominaciones: senectud, ancianidad, vejez, mayores, segunda juventud, provectos y tercera edad, tienen todos los propósitos de esquivar los prejuicios y la discriminación. Algunos de ellos sí lo logran, y en gran medida; otros, por el contrario, generan nuevas máscaras.

También los estudios sociológicos han asignado prioridades al tema y que varían en las distintas sociedades y épocas a la par de alimentación, vivienda, salud y transportes, hay abuso de los mayores como discriminación por edad en los ambientes laborales. Una consideración también respecto al "estar activo" y a las aficiones pensadas con un diseño universal, que sustituyen a los trabajos en los mayores y que también pueden ser proyectadas desde muchos años antes, por ejemplo: la lectura como recurso y como afición, que, si no ha sido ya utilizada en la juventud y edad adulta, difícilmente será utilizada mucho después. Priman los temas vitales y no quieren que estén ausentes tampoco los propios de su grupo social de personas mayores, como cultura diferente e igualmente con una diferente actitud a lo que es nivel de vida. Es muy sensible al puesto en la nueva sociedad de los mayores por la pérdida de la familia extensa y el advenimiento de la familia nuclear o sólo de pareja, con pocos hijos. Lo es también su capacidad de maniobra por el aumento de rentas y consecuente independencia de los futuros 'mayores', por el aumento de la clase media.

La tercera edad es una etapa de la vida en la que el ser humano elabora una reflexión de lo que ha logrado en su trayectoria de vida, así como de las oportunidades que se han dejado pasar o simplemente se han postergado. Cabe señalar que en esta etapa se hacen presentes los trastornos biológicos y psicológicos. Es decir, se comienza a tener problemas con la vista, la audición, el habla, el equilibrio y la pérdida de memoria.

También suele perderse a seres queridos y amigos, y la incapacidad para participar en actividades que antes solía realizar a menudo. Esto puede resultar en una desestabilidad 
emocional que da lugar a emociones negativas como la tristeza, la ansiedad, la soledad y la baja autoestima, que a su vez conducen al aislamiento social y la apatía.

La consecuencia más grave sería la depresión crónica o la depresión que es recurrente y persistente. Ésta puede tener efectos físicos que incrementan el índice de mortalidad de los hombres y mujeres. La depresión puede llevar a hábitos alimenticios que acaben resultando en obesidad, provocando también pérdida considerable del apetito y la reducción de niveles de energía, ocasionado a veces un trastorno conocido como anorexia geriátrica. Asimismo, pueden experimentar índices más altos de insomnio y tiempos de reacción más prolongados que lo normal, lo que aumenta los riesgos asociados con cocinar y conducir.

Es por esto que se debe poner un especial interés en la vida de los adultos mayores o de la tercera edad, ya que, si ellos son acompañados en esta etapa de cambios, y se sienten apoyados, se puede reducir el riesgo de padecer depresión. También si ellos ejercitan su memoria y realizan actividades, ya sean físicas o recreativas, a un ritmo moderado, pueden sentirse útiles y así motivar al grado de aumentar su autoestima

La Geriatría estudia la prevención de curación y rehabilitación de enfermedades en la tercera edad; y la Gerontología estudia los aspectos psicológicos, educativos, sociales, económicos y demográficos relacionados con los adultos mayores. Hoy en día existen especialidades como la gerontología psiquiátrica (o psiquiatría gerontológica), que incluyen variables como la calidad de vida y otros fundamentos científicos. También existe la educación gerontológica, que tiene como finalidad orientar los preceptos pedagógicos a la ciencia de la gerontología para enriquecerla y reforzarla. Se ha desarrollado también como una tecnología social como apoyo e industrialización como de avances científicos. El Día Internacional de las Personas de Edad se celebra el 1 de octubre.

La hipertensión arterial (HTA) es una enfermedad crónica caracterizada por un incremento continuo de las cifras de la presión sanguínea en las arterias. Aunque no hay un umbral estricto que permita definir el límite entre el riesgo y la seguridad, de acuerdo con consensos internacionales, una presión sistólica sostenida por encima de $139 \mathrm{mmHg}$ o una presión diastólica sostenida mayor de $89 \mathrm{mmHg}$, están asociadas con un aumento medible del riesgo de aterosclerosis $\mathrm{y}$, por lo tanto, se considera como una hipertensión clínicamente significativa. [1]

La hipertensión arterial se asocia a tasas de morbilidad y mortalidad considerablemente elevadas, por lo que se considera uno de los problemas más importantes de salud pública, especialmente en el adulto mayor. La hipertensión es una enfermedad asintomática y fácil de detectar; sin embargo, cursa con complicaciones graves y letales si no se trata a tiempo. La hipertensión crónica es el factor de riesgo modificable más importante para desarrollar enfermedades cardiovasculares, así como para la enfermedad cerebrovascular y renal.[2] Se sabe también que los hombres tienen más predisposición a desarrollar hipertensión arterial 
que las mujeres, situación que se modifica cuando la mujer llega a la menopausia, ya que antes de esta posee hormonas protectoras que desaparecen en este periodo, a partir de ese momento la frecuencia se iguala, por lo tanto la mujer debe ser más vigilada para esta enfermedad en los años posteriores de la menopausia.

La hipertensión arterial, de manera silente, produce cambios en el flujo sanguíneo, a nivel macro y microvascular, causados a su vez por disfunción de la capa interna de los vasos sanguíneos y el remodelado de la pared de las arteriolas de resistencia, que son las responsables de mantener el tono vascular periférico. Muchos de estos cambios anteceden en el tiempo a la elevación de la presión arterial y producen lesiones orgánicas específicas.

Los diuréticos y los betabloqueantes reducen la aparición de eventos adversos por hipertensión arterial relacionados con la enfermedad cerebrovascular. Sin embargo, los diuréticos son más eficaces en la reducción de eventos relacionados con la enfermedad cardíaca coronaria. Los pacientes hipertensos que cumplen su tratamiento tienen menos probabilidades de desarrollar hipertensión grave o insuficiencia cardíaca congestiva. [4] En la mayoría de los casos, en los ancianos se utilizan dosis bajas de diuréticos como terapia inicial antihipertensiva. En pacientes ancianos con hipertensión sistólica aislada suele utilizarse como terapia alternativa un inhibidor de los canales de calcio de acción prolongada, tipo dihidropiridina

Algunos de los factores ambientales que contribuyen al desarrollo de la hipertensión arterial incluyen la obesidad, el consumo de alcohol, el tamaño de la familia, circunstancias de nacimiento y las profesiones estresantes. Se ha notado que, en sociedades económicamente prósperas, estos factores aumentan la incidencia de hipertensión con la edad.

Al transcurrir los años y según los aspectos de la enfermedad, el número de fibras de colágeno en las paredes arteriales aumenta, haciendo que los vasos sanguíneos se vuelvan más rígidos. Al reducirse así la elasticidad, el área seccional del vaso se reduce, creando resistencia al flujo sanguíneo y como consecuencia compensadora, se aumenta la presión arterial.

Si bien no es posible eliminar por completo la hipertensión, varias acciones son muy útiles y necesarias para prevenir su aparición y para evitar el empeoramiento de los síntomas:

- Incrementar la actividad física aeróbica;

- Mantener un peso corporal dentro de los estándares correspondientes a la edad y la estatura, según el sexo

- Reducir al mínimo el consumo de alcohol

- Consumir una dieta rica en frutas y vegetales 
- Privarse de todo tipo de tabaco

\section{Sistema de ejercicios para hipertensos de la tercera edad:}

- Articulación del cuello: flexión lateral, flexión hacia el frente, torsión del cuello, semicírculo sin llevarlo hacia la parte trasera de la cabeza y a un ritmo lento para evitar mareo en los ancianos. No se debe realizar la flexión hacia atrás

- Brazos: círculos hacia el frente y hacia atrás, extensión arriba y abajo, cruzamiento y extensión lateral y abducción de estos

- Codo: flexión y extensión

- Mano: movimientos laterales hacia la derecha o la izquierda, flexión palmar-dorsal y palmar-ventral.

- Dedos: flexión y extensión de estos

- Tronco: flexión lateral, ya sea hacia la derecha o la izquierda, torsiones laterales de igual forma, ligeras flexiones ventrales y no se deben hacer flexiones dorsales

- Piernas: semiflexión y extensión de la pierna en conjunto

- Pie: flexión dorsal, movimientos laterales, en círculo y flexión plantar y ventral

\section{Ejercicios de fuerza:}

- Planchas contra la pared, trabajo con peso pequeño (flexión y extensión de los brazos con peso, abducción con peso), semicuclillas y elevación de los talones con apoyo sobre el metatarso

\section{Ejercicios de equilibrio:}

- Apoyo sobre un solo pie mientras el otro se rota por distintas posiciones con apoyo, apoyo de los talones o de los metatarsos ayudados de una pared o persona, desplazamiento sobre una franja dibujada en el piso

\section{Ejercicios de resistencia:}

- Realizar caminatas con diferentes ritmos y un trote moderado

- Todos estos ejercicios deben realizarse manteniendo un nivel de intensidad entre un 60\%-65\% de su frecuencia cardiaca máxima. La toma de la FC debe ser 4 veces durante la práctica de ejercicios: 1 . Antes del calentamiento, 2. Después de $1 \mathrm{~min}$ del 
calentamiento, 3. Después de terminar los ejercicios, 4. Después de la recuperación completa o a la salida

\section{Conclusiones.}

- En la actualidad, los esfuerzos por combatir la discriminación del anciano rinden frutos gracias a la creciente notoriedad de adultos mayores sanos y activos. En nuestro país se realizan actividades recreativas y ejercicios físicos, los cuales a través de estos se logra romper con la rutina y con el sedentarismo, contribuyendo a que estas personas tengan un envejecimiento lo más saludable posible. Si bien no es posible eliminar por completo la hipertensión, varias acciones son muy útiles y necesarias para prevenir su aparición y para evitar el empeoramiento de los síntomas.

\section{Referencias bibliográficas.}

2.02.12.22.32.4Dreisbach, Albert W; SatSharma y Claude Kortas (feb de 2010). «Hypertension» (en inglés). Nephrology: Hypertension and theKidney. eMedicine.com. Consultado el 5 de julio de 2010.

José F. Guadalajara Boo (jefe de Servicio Clínico del Instituto Nacional de Cardiología Ignacio Chávez). Programa de actualización continúa para Cardiología. pp. p8.

4.04.1 DÍAZ, Mónica. ¿Cómo comenzar el tratamiento del paciente hipertenso?.Revista Argentina de Cardiología [online]. 2006, vol.74, n.3 [citado 2010-07-23], pp. 191193. ISSN 1850-3748.

Sigerist, Henry E. (1965). GroßeÄerzte. Múnich (Alemania): J. F. LehmansVerlag (5. auflage) (1. auflage 1958). plate 26, pág. 120.

Girish DWIVEDI y ShridharDwivedi (2007): «'History of Medicine: Sushruta, theclinicianteacherparexcellence», enNationalInformatics Centre (Gobierno de India).

7.07.17.27.37.47.5Esunge PM (octubre 1991). «From blood pressure to hypertension: the history of research». J. R. Soc. Med.84 (10): pp. 621. PMID 1744849.

edited by J.D. Swales. (1995). Manual of hypertension. Oxford: BlackwellScience. pp. xiii. ISBN 0-86542-861-1.

edited by J.D. Swales. (1995). Manual of hypertension. Oxford: BlackwellScience. pp. xiii. ISBN 0-86542-861-1.

Harrison Principios de Medicina Interna 16a edición (2006). «Capítulo 230. Vasculopatía hipertensiva» (en español). Harrison online en español. McGraw-Hill. Consultado el 16 de junio de 2008. 
11.011.111.211.311.411.511.6Chobanian AV, Bakris GL, Black HR, et al. (December 2003). «Seventh report of the Joint National Committee on Prevention, Detection, Evaluation, and Treatment of High Blood Pressure». Hypertension42 (6): pp. 120652. doi:10.1161/01.HYP.0000107251.49515.c2. PMID 14656957.

12.012.1Mancia G, De Backer G, Dominiczack A, et al. (2007). «2007 Guidelines for the management of arterial hypertension» (eninglés). European Heart Journal (28): pp. 1462-1536. Archivado. Plantilla:Citation error.

13.013.1Mancia G, De Backer G, Dominiczack A, et al. (2007). «Guías de práctica clínica para el tratamiento de la hipertensión arterial 2007» (en español). RevEspCardiol.9 (60): pp. 968.e1-e94.

14.014.114.2 «Hypertension: clinical management of primary hypertension in adults». NationalInstituteforHealth and ClinicalExcellence. Consultado el 30-12-2012.

Mancia G, Bertinieri G, Grassi G, Parati G, Pomidosi G, Ferrari A, et al. Effects of bloodpressure measurements by the doctor on patient`s blood pressure and heart rate. Lancet. 1983.;2:695-8

Calhoun DA, Jones D, Textor S, et al.(June 2008). «Resistant hypertension: diagnosis, evaluation, and treatment. A scientific statement from the American Heart Association Professional Education Committee of the Council for High Blood Pressure Research». Hypertension51 (6): pp. 1403-19. doi:10.1161/HYPERTENSIONAHA.108.189141. PMID 18391085.

«CG34 Hypertension - quick reference guide» (PDF). National Institute for Health and Clinical Excellence (28 de junio de 2006). Consultado el 04-03-2009.

Jetté M, Landry F, Blümchen G (April 1987). «Exercise hypertension in healthy normotensive subjects. Implications, evaluation and interpretation». Herz12 (2): pp. 110-8. PMID 3583204.

Pickering TG (April 1987). «Pathophysiology of exercise hypertension». Herz12 (2): pp. 119-24. PMID 2953661.

20.020.1Rost R, Heck H (April 1987). «[Exercise hypertension--significance from the viewpoint of sports]» (en alemán). Herz12 (2): pp. 125-33. PMID 3583205.

21.021.1Klaus D (April 1987). «[Differential therapy of exercise hypertension]» (en alemán). Herz12 (2): pp. 146-55. PMID 3583208.

Mancia G, Bertinieri G, Grassi G, Parati G, Pomidosi G, Ferrari A, et al.: «Effects of bloodpressure measurements by the doctor on patient's blood pressure and heart rate», en Lancet, 1983; 2: págs. 695-698 
Ticinesi, Andrea; Nouvenne, Antonio; Maalouf, Naim M; Borghi, Loris; Meschi, Tiziana (2014). «Salt and nephrolithiasis». NephrologyDialysisTransplantation.

http://www.jstage.jst.go.jp/article/jphs/100/5/370/_pdf A Missing Link Between a High Salt Intake and Blood Pressure Increase: Makoto Katori and MasatakaMajima, Department of Pharmacology, Kitasato University School of Medicine, Kitasato, Sagamihara, Kanagawa, Japan February 8, 2006

Silverberg DS, Iaina A and Oksenberg A (January 2002). «Treating Obstructive Sleep Apnea Improves Essential Hypertension and Quality of Life». American FamilyPhysicians65 (2): pp. 229-36. PMID 11820487.

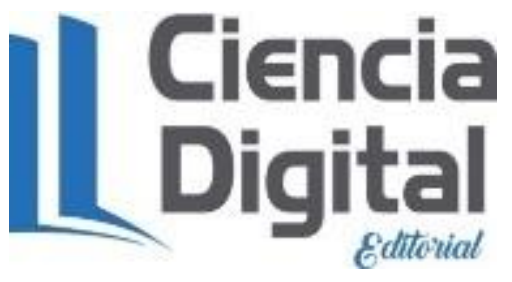


PARA CITAR EL ARTÍCULO INDEXADO.

Mederos González, A., \& Rojas Puig, J. F. (2019). El adulto mayor en la sociedad. Anatomía Digital, 2(2), 84-92. https://doi.org/10.33262/anatomiadigital.v2i2.1082

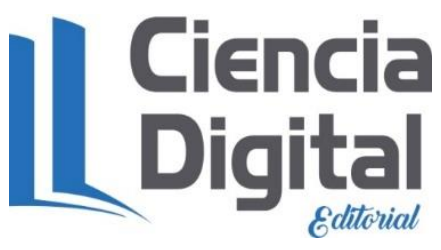

El artículo que se publica es de exclusiva responsabilidad de los autores y no necesariamente reflejan el pensamiento de la Revista Anatomía Digital.

El artículo queda en propiedad de la revista y, por tanto, su publicación parcial y/o total en otro medio tiene que ser autorizado por el director de la Revista Anatomía Digital.
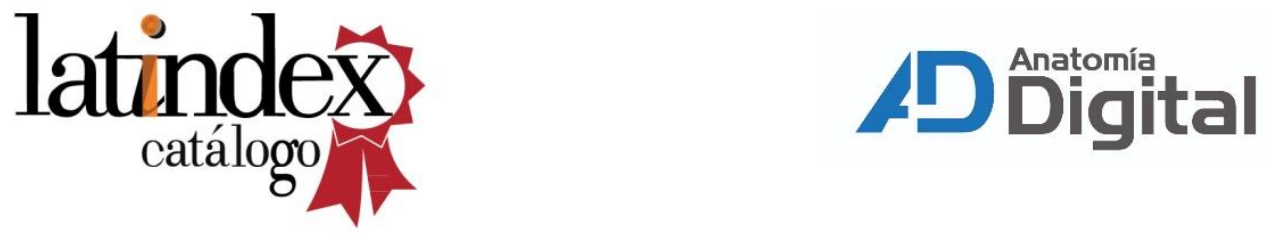